\title{
Characterization of ex vivo healthy human axillary lymph nodes with high resolution 7 Tesla MRI
}

\author{
M. A. Korteweg • J. J. M. Zwanenburg • P. J. van Diest • M. A. A. J. van den Bosch • \\ P. R. Luijten • R. van Hillegersberg • W. P. Th. M. Mali • W. B. Veldhuis
}

Received: 22 March 2010 /Revised: 18 June 2010 / Accepted: 21 July 2010 / Published online: 9 August 2010

(C) The Author(s) 2010. This article is published with open access at Springerlink.com

\begin{abstract}
Objective To characterize ex vivo healthy human axillary lymph nodes on 7 Tesla MRI and to correlate the findings with pathological analysis as a first step towards non-invasive staging of breast cancer patients in the future.

Methods Four axillary lymph node dissection (ALND) specimens from 2 autopsy patients, who had no cancer, were examined on a clinical 7 Tesla MRI system. For morphological analysis a 3D T1-weighted fat-suppressed fast-field-echo [isotropic resolution $180 \mu \mathrm{m}$ ] was acquired. For quantitative analyses 2D T1-, 3D T2-, T2*- and diffusion-weighted images were acquired. The ALNDs were mapped and stained for precise correlation of MRI to pathology. Nodes were sliced in $3 \mu \mathrm{m}$ sections, Haematoxylin \& Eosin stained, and examined by an experienced pathologist.

Results MRI detected all 45 nodes and 6 additional nodes that were not detected at pathological analysis. B-cell follicles, efferent- and afferent lymph vessels and blood vessels were identified. Mean T1, T2, T2*, ADC values
\end{abstract}

M. A. Korteweg $(\bowtie) \cdot$ J. J. M. Zwanenburg •

M. A. A. J. van den Bosch - P. R. Luijten - W. P. T. M. Mali •

W. B. Veldhuis

Department of Radiology, University Medical Centre Utrecht, HP.E01.102, Heidelberglaan 100,

3584 CX Utrecht, The Netherlands

e-mail: mieskorteweg@gmail.com

\section{P. J. van Diest}

Department of Pathology, University Medical Centre Utrecht,

Room E04.312; Heidelberglaan 100,

3584 CX Utrecht, The Netherlands

\section{R. van Hillegersberg}

Department of Surgery, University Medical Centre Utrecht,

Room G04.228; Heidelberglaan 100,

3584 CX Utrecht, The Netherlands
( \pm standard deviation) were $944 \pm 113 \mathrm{~ms}, 32 \pm 2 \mathrm{~ms}, 16 \pm$ $2 \mathrm{~ms}, 0.39 \pm 0.09 \cdot 10^{-3} \mathrm{~mm}^{2} / \mathrm{s}$, respectively.

Conclusions 7 Tesla MRI of ex vivo human axillary lymph nodes correlated well with pathology. MRI detected all nodes present in the specimens and allowed visualization of fine structural detail. Pathology-correlated quantitative MRI data are presented.

Keywords Magnetic resonance imaging .

Breast neoplasms $\cdot$ Lymph nodes $\cdot$ Neoplasm staging .

7 Tesla

\section{Introduction}

Breast cancer is the most common form of cancer in women worldwide [1]. The most important factor determining prognosis for these patients is axillary lymph node status $[2,3]$. Assessment of nodal status currently requires surgery, by performing a sentinel node procedure, followed by an axillary dissection in case of a positive sentinel node [4]. This procedure can cause morbidity and therefore, less invasive staging procedures, such as by imaging, are warranted $[5,6]$.

Diffusion weighted MRI (DWI) has shown promise in the detection of lymph nodes, but so far cannot accurately discriminate benign from malignant nodes [7,8]. With the use of ultra-small particles of iron oxide (USPIOs), MRI has shown to be able to assess axillary nodal status in vivo with a high positive and negative predictive value [9]. However, this contrast-agent has not been approved for clinical application yet, and identification of metastatic lymph nodes, based solely on morphological criteria, remains challenging [10-12].

Recently, MRI systems with a magnetic field strength of 7 Tesla (T) have come into use. We have used a whole body 
7T MRI platform to determine morphological and quantitative characteristics, such as T1, T2, T2* and apparent diffusion coefficients (ADC), ex vivo of healthy human axillary lymph nodes. Knowledge of benign lymph node characteristics may support a better assessment of metastatic nodes in vivo in the future. MRI data was correlated to the gold standard, pathology, on a node to node basis.

\section{Material and methods}

\section{Harvesting of lymph nodes}

During two autopsies, four axillary lymph node dissection (ALND) specimens were retrieved. The deceased were two females who died of cardiovascular disease and who had donated their bodies to science. No breast- or other cancers were detected. An ALND is a block of fatty tissue in which the axillary lymph nodes are located; nodal configuration and peri-nodal anatomy is thus maintained. Subsequently, the specimens were stitched onto a plastic grid and were fixed in formaldehyde for at least 1 week (range 1 week11 weeks).

\section{MRI protocols}

The specimens were examined using a commercial 7T MRI system (Philips Healthcare, Cleveland, USA), using a transmit/receive head coil with a 16 channel receive coil (Nova Medical, Wilmington, MA, USA). During MR data acquisition, the specimens were submersed in fomblin (Solvay Solexis, Bollate, Italy) to provide susceptibility matching [13].

The MR-protocol for all ALNDs (51 nodes) consisted of:

1) A $3 \mathrm{D} \mathrm{T} 1$ weighted (3D $\mathrm{T} 1 \mathrm{~W})$ fat-suppressed fast field echo [TR/TE $158 / 4.8 \mathrm{~ms}$, flip angle $35^{\circ}$, FOV $23.6 \times$ $110 \times 110 \mathrm{~mm}^{3}$, acquired resolution $180 \mu \mathrm{m}$ isotropic] (acquisition time; $5 \mathrm{~h}$ and $10 \mathrm{~min}$ ),

2) A T1-map which was obtained with a $2 \mathrm{D}$ Look-Locker sequence [TR between inversions $4,000 \mathrm{~ms}$, TR/TE $7.6 / 3.7 \mathrm{~ms}$, flip angle $3^{\circ}$, FOV $110 \times 110 \mathrm{~mm}^{2}$, in-plane acquired resolution $500 \times 500{\mu \mathrm{m}^{2}}^{2}$, slice thickness $5 \mathrm{~mm}, 100$ samples with an initial TI of $18 \mathrm{~ms}$ and increments of $40 \mathrm{~ms}$ per sample] (acquisition time $35 \mathrm{~min})$.

For ALND 3 and 4 (34 nodes) the following quantitative data sets were also acquired:

3) A multi-slice T2-map which was obtained from a multi-echo spin echo sequence $[\mathrm{TR} /$ first TE/ $\Delta \mathrm{TE} 800 /$ $6 / 6 \mathrm{~ms}, 15$ echoes, FOV $24 \times 110 \times 110 \mathrm{~mm}^{3}$, resolution $500 \mu \mathrm{m}$ isotropic] (acquisition time $5 \mathrm{~h}$ and $42 \mathrm{~min}$ ),
4) A multi-slice $T 2 *$-map which was obtained from a multi-echo gradient echo sequence $[\mathrm{TR} /$ first $\mathrm{TE} / \Delta \mathrm{TE}$ 300/5.9/5.9 ms, 10 echoes, flip angle $35^{\circ}$, FOV $24 \times$ $110 \times 110 \mathrm{~mm}^{3}$, resolution $350 \mu \mathrm{m}$ isotropic] (acquisition time $2 \mathrm{~h}$ and $47 \mathrm{~min}$ ),

5) A multi-slice diffusion weighted image [TR/TE 4000/ $83 \mathrm{~ms}$, FOV $24 \times 110 \times 110 \mathrm{~mm}^{3}$, resolution $1.0 \mathrm{~mm}$ isotropic, b-values 0,750 and 1,500 $\mathrm{s} / \mathrm{mm}^{2}$ ] (acquisition time $2 \mathrm{~h}$ and $18 \mathrm{~min}$ ).

Pathology

Pathological examination was performed according to standard ALND protocol by an experienced pathologist, who was blinded to the MRI results during the initial evaluation of the specimen. Detected nodes were sliced into $4 \mathrm{~mm}$ sections and were paraffin embedded after which $3 \mu \mathrm{m}$ thick slices were cut from each $4 \mathrm{~mm}$ block, which were subsequently stained with Haematoxylin \& Eosin (H\&E). Following standard pathological examination, the ALND specimen was correlated to the MRI-based map to identify any remaining nodes. After removal of any remaining MRI-detected nodes, the residual fatty tissue was submersed in acetone to detect any small lymph nodes which might have been overlooked. For matching purposes with MRI, digital pictures of all sections were obtained at a 1.5 magnification with a digital camera (Leica, Rijswijk, the Netherlands) mounted on top of a standard microscope (Leica).

MRI to pathology correlation

After positioning on the plastic grid, a detailed map was drawn of each specimen, noting the location of the individual nodes as they were situated in the fatty ALND tissue, using the horizontal and vertical lines of the grid as longitude and latitude references. Nodes detected by the pathologist were visually matched to nodes on this map. After removal of each node, it was dyed to permanently mark the sides which were superior and left on the MR images.

The high resolution 3D T1W protocol (voxel volume 5.8 nanoliter) allowed reconstruction along arbitrary planes which enabled accurate matching of the MR images to the sectioning plane of microscopic pathology slides.

\section{MRI analysis}

Quantitative and morphologic MRI analysis was done using in-house software created in MATLAB (Mathworks, USA). The mean absolute $\mathrm{T} 1$ relaxation times were determined for all nodes in all specimens. Quanti- 
tative analysis of $\mathrm{T} 2$ and $\mathrm{T} 2 *$ relaxation times and the ADCs were determined. Values were determined by drawing ROIs on the transversal views of the fitted maps derived from the original images. The T1 values were calculated according to a T1-mapping acquisition method described by Hsu et al. [14]. The "Hsu algorithm" was selected after testing it on our samples against a 3 parameter non-linear least-squares algorithm which fitted the data directly to the Look-Locker equation. The Hsu-method correlated better to "gold standard" MR spectroscopy $\mathrm{T} 1$ measurements performed on the same specimens (data not shown). For T2-mapping only the even echo times were used to minimize the sensitivity to errors in the pulse angle of the 180 degrees refocusing pulses.

In addition to the measured $\mathrm{T} 1$ values, temperaturecorrected $\mathrm{T} 1$ values are presented, which were calculated according to Rieke et al. [15], who described an increase in T1 values of approximately $1.5 \%$ per degree Celsius. Similarly, ADC measured at room temperature, was extrapolated to body temperature, assuming a linear relationship, according to the Stokes-Einstein equation.

Morphologic analysis was performed on the high resolution 3D T1W MR data sets. The longest diameter (length) and the perpendicular in-plane diameter (width) were measured on transversal images. The shape, presence of a fatty centre and detailed structural architecture of the nodes were described.

All quantitative results were expressed as means with a standard deviation. A correlation of the size and the morphologic features was performed using Spearman's rank test.

\section{Results}

MRI detected 51 nodes in the four ALNDs. Figure 1 shows a maximum intensity projection of the 3D T1W data set of specimen 2. Forty-two of 51 nodes $(82 \%)$ were retrieved during routine dissection of the ALNDs. Of the remaining nine MRI-detected nodes, three could be retrieved on reexamination of the specimen; one directly after consulting the MRI-based map and two others after defatting the specimen with acetone. The remaining six MRI-detected nodes -all smaller than $3 \times 2 \mathrm{~mm}^{2}$ - were not found at pathological examination.

\section{Quantitative analysis}

Table 1 shows the number of nodes detected by MRI and by pathology and the various MRI characteristics per ALND. The influence of conservation time on T1, T2 and T2* is shown in Fig. 2.

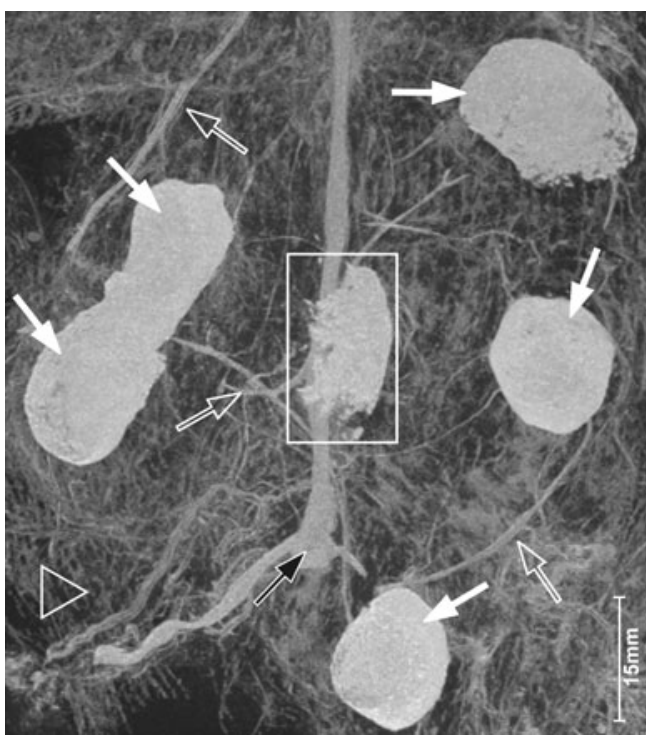

Fig. 1 Thin-slab maximum intensity projection over $2 \mathrm{~mm}$ of a 3D T1W MR data set (180 $\mu \mathrm{m}$ isotropic resolution) of an axillary lymph node dissection specimen. Solid white arrows: Lymph nodes depicted against a black background consisting of suppressed fat. Open white arrows: Lymph- and blood vessels. Black arrow: A macroscopically proven blood vessel. Arrowhead: An artifact caused by a gauze, which had not been drenched in fomblin. Rectangle: A remnant of formaldehyde inside the pleats of the specimen

Lymph node $\mathrm{T} 1$ values, determined at a room temperature of $20^{\circ} \mathrm{C}$, were: $931 \mathrm{~ms}, 806 \mathrm{~ms}, 910 \mathrm{~ms}$ and $1,049 \mathrm{~ms}$ for ALND 1, 2, 3 and 4 respectively, leading to an overall average T1 of $944 \mathrm{~ms}$. The body-temperature-corrected T1 values for ALND 1, 2, 3 and 4 are 1,168 ms, 1,011 ms, $1,140 \mathrm{~ms}$ and $1,316 \mathrm{~ms}$ respectively, leading to an overall average $\mathrm{T} 1 \mathrm{of} 1,316 \mathrm{~ms}$.

Lymph node ADC values, determined at a room temperature of $20^{\circ} \mathrm{C}$, were: $0.33 \cdot 10^{-3} \mathrm{~mm}^{2} / \mathrm{s}$ and $0.45 \cdot 10^{-3} \mathrm{~mm}^{2} / \mathrm{s}$ for ALND 3 and 4 respectively, leading to an overall average ADC of $0.39 \cdot 10^{-3} \mathrm{~mm}^{2} / \mathrm{s}$. The bodytemperature-corrected $\mathrm{ADC}$ values result in an average ADC for ALND 3 and 4 of $0.35 \cdot 10^{-3} \mathrm{~mm}^{2} / \mathrm{s}$ and $0.47 \cdot 10^{-3} \mathrm{~mm}^{2} / \mathrm{s}$ respectively, leading to an overall average ADC of $0.41 \cdot 10^{-3} \mathrm{~mm}^{2} / \mathrm{s}$.

\section{Morphological analysis}

Twenty-three of 45 nodes (51\%) were observed without a lipid-rich medulla (Fig. 3). The Spearman's rank correlation ratio for length, width and length/width (i.e. the roundness) of the node correlated to the presence of a lipid centre, was 0.54 for length ( $p=0.0001$ [95\% CI $0.32-0.71]$ ), 0.54 for width $(p=0.0001 \quad[95 \%$ CI $0.31-0.71])$ and 0.031 for roundness ( $p=0.83$ [95\% CI $-0.25-0.31]$ ).

Lymph nodes are connected to lymphatic pathways and blood vessels. Lymph is conducted to the node 
Table 1 Lymph node characteristics depicted per axillary lymph node dissection (ALND)

\begin{tabular}{|c|c|c|c|c|c|}
\hline & ALND 1 & ALND 2 & ALND 3 & ALND 4 & All ALNDs \\
\hline$\#$ of nodes at MRI & 9 & 8 & 15 & 19 & 51 \\
\hline$\#$ of nodes at pathology & 9 & 8 & 12 & 16 & 45 \\
\hline Length $( \pm \mathrm{SD})$ [range]mm & 11 (6) [2.7-19.1] & $10(7)[1.3-21.6]$ & $9(8)[2.1-27.2]$ & 9 (7) [3.0-24.1] & 9 (7) [1.3-27.2] \\
\hline Width $( \pm \mathrm{SD})$ [range]mm & 7 (5) [1.9-13.2] & 7 (6) $[0.6-15.8]$ & $6(5)[1.3-17.0]$ & 5 (4) $[1.9-13.0]$ & $6(5)[0.6-17.0]$ \\
\hline $\mathrm{T} 1( \pm \mathrm{SD}) \mathrm{ms}$ & $931(87)$ & $806(52)$ & $910(39)$ & $1,049(81)$ & $944(113)$ \\
\hline $\mathrm{T} 2 *( \pm \mathrm{SD}) \mathrm{ms}$ & - & - & $15(1)$ & $17(3)$ & $16(2)$ \\
\hline $\mathrm{T} 2( \pm \mathrm{SD}) \mathrm{ms}$ & - & - & $31(2)$ & $34(1)$ & $32(2)$ \\
\hline $\mathrm{ADC}( \pm \mathrm{SD}) \mathrm{mm}^{2} / \mathrm{s}$ & - & - & $0.33 \cdot 10^{-3}(0.1)$ & $0.45 \cdot 10^{-3}(0.1)$ & $0.39 \cdot 10^{-3}(0.09)$ \\
\hline
\end{tabular}

The total number (\#) of nodes found on MRI and by pathology, the mean transversal dimensions in millimeters (mm) \pm standard deviation (SD) and range, the mean $\mathrm{T} 1, \mathrm{~T} 2$ and $\mathrm{T} 2 *$ relaxation times and the mean apparent diffusion coefficient (ADC) are depicted

through afferent vessels on the convex side. The lymph exits the node through efferent vessels on the concave hilar side, together with the vasculature [16]. Typically, there are more afferent than efferent vessels [16]. We visualized many small afferent vessels at nodal convexities and a few, often prominent, efferent vessels exiting the node from the hilus (Fig. 4). The lymph node capsule contains a vascular network which runs parallel to the lymph flow in order to facilitate the exchange of fluid components. These vascular structures can become dilated, which was visualized in Figs. 3f, 5a and 6b.

The outer part of the lymph node capsule contains centres called B-cell follicles, which mainly consist of B-cell lymphocytes; These centres are described as either primary (inactive), or secondary (activated) [16]. On 3D T1W MRI several hypo-intense regions were depicted in the hyper-intense capsule as shown in Figs. 4a and $6 \mathrm{a}$. These structures visualized on MRI corresponded to the location of activated B-cell follicles seen at pathology. At pathology these centres had diameters of up to $1.3 \mathrm{~mm}$ (range $0.1-1.3 \mathrm{~mm}$ ). On MRI the diameters ranged from 0.4 to $1.5 \mathrm{~mm}$.

\section{Discussion}

We presented the $7 \mathrm{~T}$ characterization of ex vivo healthy human axillary lymph nodes with a node-to node correlation to pathology. 45 axillary nodes from 4 ALNDs were characterized on MRI and subsequently correlated to pathology. This study is an ex vivo study in which normal lymph node characteristics were assessed at $7 \mathrm{~T}$. Therefore lengthy MR protocols could be used for this purpose. However this can not be applied to in vivo characterization of nodes. Nonetheless, taking technological advancements into consideration as well, knowledge gained by analyzing ex vivo nodes can help with a quicker assessment in the future of lymph nodes in vivo in cancer patients.

Nodal detection rate was higher for MRI as compared to pathology

Previously Luciani et al. performed an ex vivo, node to node correlation study at $1.5 \mathrm{~T}$ showing that MRI accurately assessed lymph node size, but missed on average $20 \%$ of the nodes found by pathology [10]. By comparison, in this
Fig. $2 \mathrm{~T} 1, \mathrm{~T} 2$ and $\mathrm{T} 2 *$ values (ms) as a function of formaldehyde fixation time per axillary lymph node dissection (ALND). The $\mathrm{T} 1$ values are depicted by "-", the $\mathrm{T} 2$ values by " $\boldsymbol{\Delta}$ " and the $\mathrm{T} 2$ * values by "»"

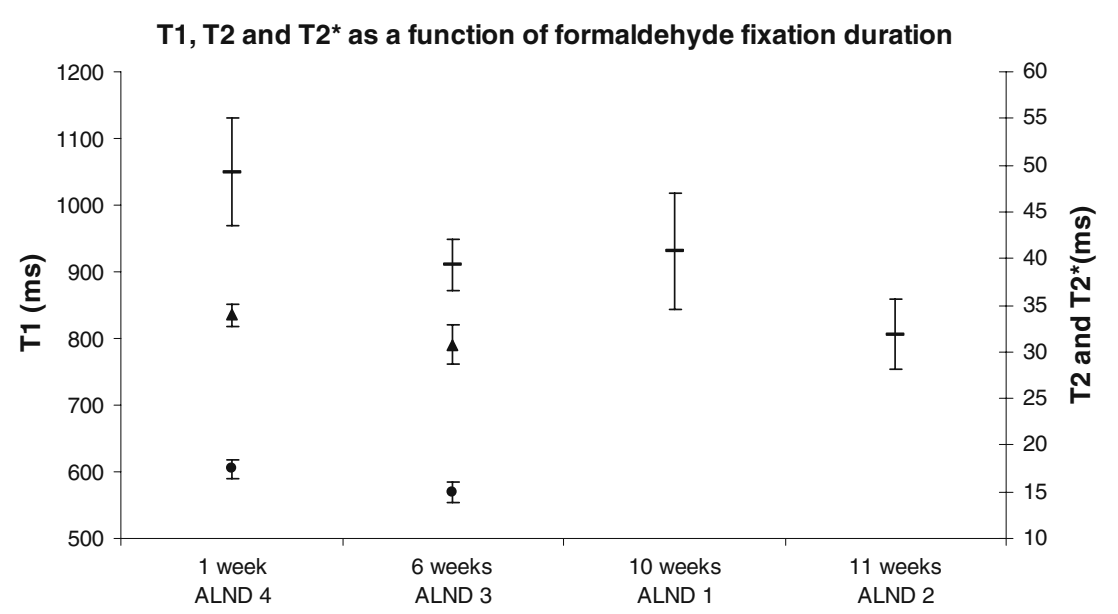


Fig. 3 Six lymph nodes on 3D T1W images $(180 \mu \mathrm{m}$ isotropic resolution) and on matching H\&E stained histopathology images (1.5× magnification). Solid thin arrows: Afferent lymph vessels, which conduct lymph towards the convex side of the node. Open arrows: Efferent lymph vessels, which exit the node on the concave side. Fat short arrow: The centre of the hilus, which is of low signal intensity on this MR image. This is most likely due to the presence of fat, which is suppressed and due to the presence of blood vessels. These are also of low signal intensity on MRI due to the high susceptibility caused by the iron (hemoglobin) content of the erythrocytes in these vessels. Open arrow head: Intranodal dilated blood vessels. The vessels contain erythrocytes on the pathology slides. These vessels have low signal intensity on the MRI scans, due to susceptibility artifacts because of the hemoglobin, iron, content. a-c Bean shaped nodes with fatty centres and a highly cellular capsule. $\mathbf{d}-\mathbf{f}$ Compact spherical nodes with no fatty centres
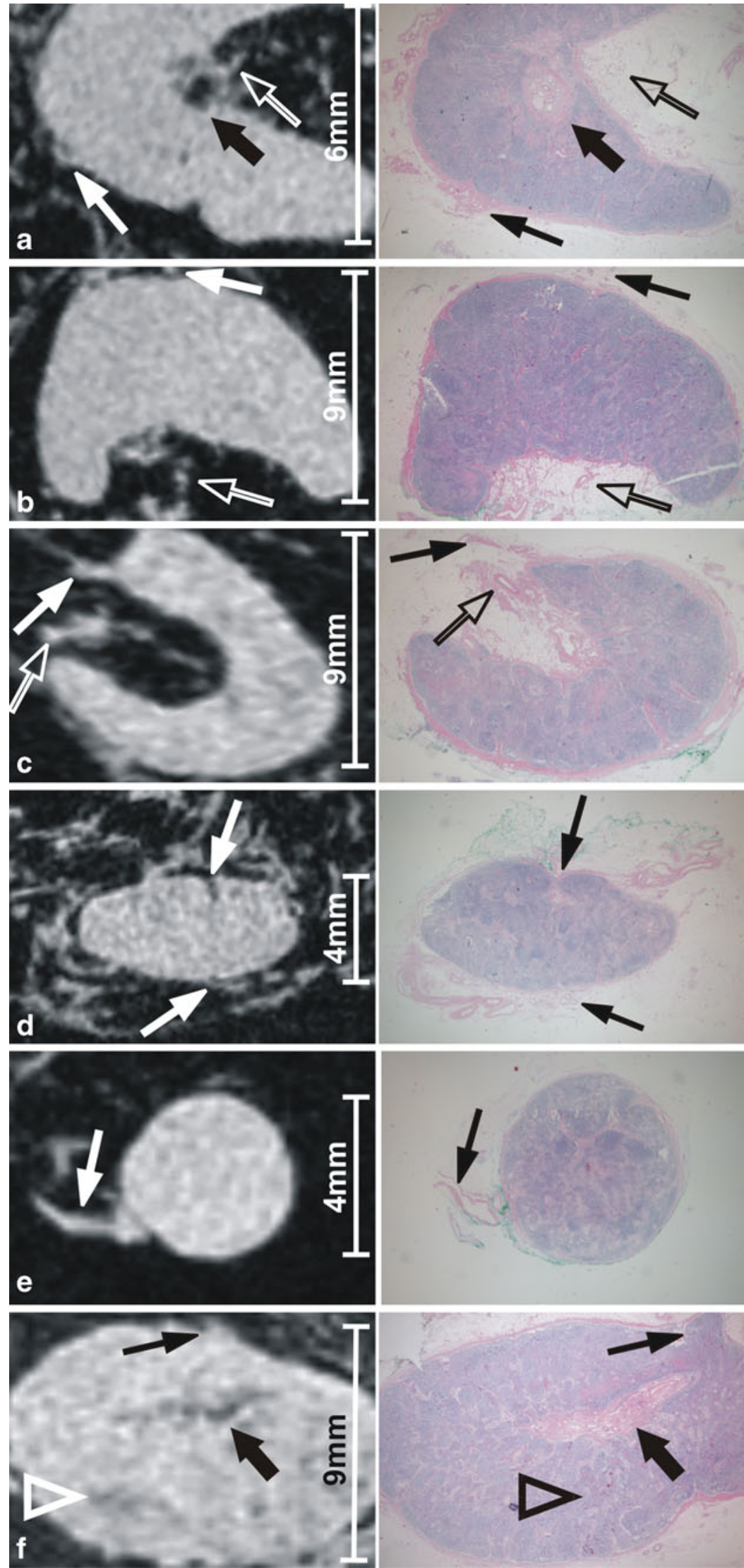

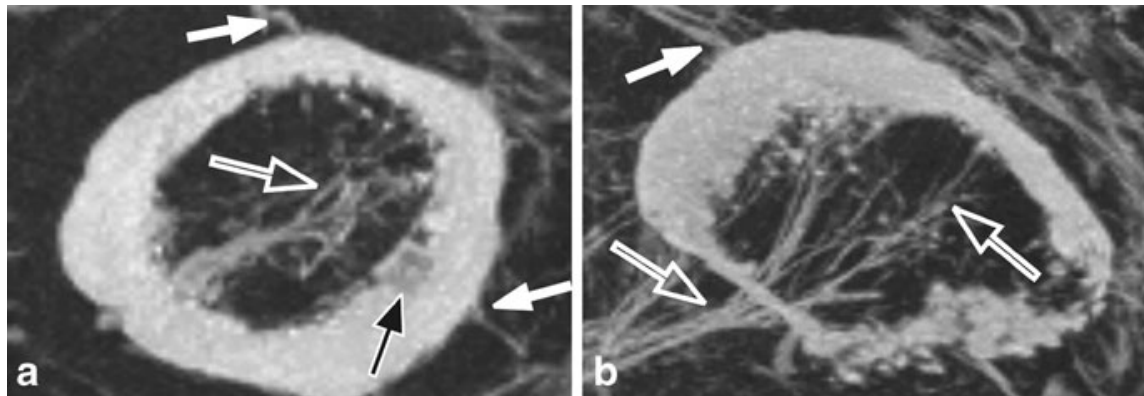

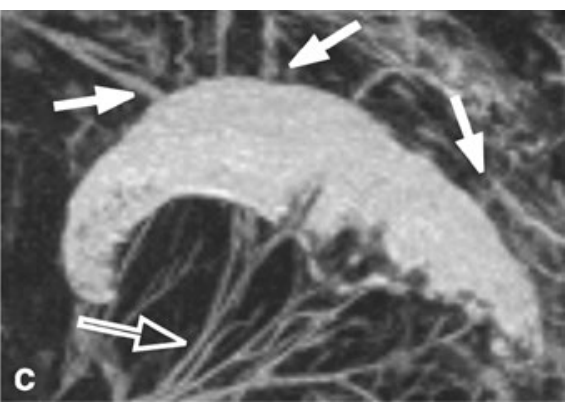

Fig. 4 The hilus of three different nodes on thin-slab maximum intensity projections (MIP) over $2 \mathrm{~mm}$ of a 3D T1W MR image (180 $\mu \mathrm{m}$ isotropic resolution). Solid white arrows: Afferent lymphatic vessels. Open arrows: Efferent lymph vessels. Thin black arrow: Bcell follicle. a An area of low signal intensity is visible in the lymph

ex vivo study, at $7 \mathrm{~T}$ we detected all nodes found at subsequent pathological examination. A likely explanation is the higher imaging-resolution -we used a 55-times smaller voxel size (voxel volume 5.8 nanoliter)- and the higher lymph node to background contrast of the fatsuppressed 3D T1W data set we acquired. Moreover, MRI node capsule. This corresponds to a B-cell follicle at pathological examination. b The MIP is oriented in such a way as to depict all efferent vessels and as many afferent vessels as possible. Not all afferent vessels can be seen in one view

detected nine more nodes than were retrieved at subsequent standard pathological examination of the ALNDs. Six of these nine MRI-detected nodes could not be correlated to pathology. Therefore in theory these would have to be considered false positives when using pathology as the gold standard. However their morphological appearance and
Fig. 5 Three lymph nodes on 3D T1W images $(180 \mu \mathrm{m}$ isotropic resolution) and on matching $H \& E$ stained histopathology images (1.5× magnification). Solid arrows: Afferent lymph vessels. Open arrows: Efferent vessels. Open arrowhead: Intranodal dilated blood vessels. a A partial view of a compact spherical node with no fatty centre. b A vessel (encircled) traverses the capsule of the node. $\mathbf{b}-\mathbf{c}$ Bean shaped nodes with fatty centres
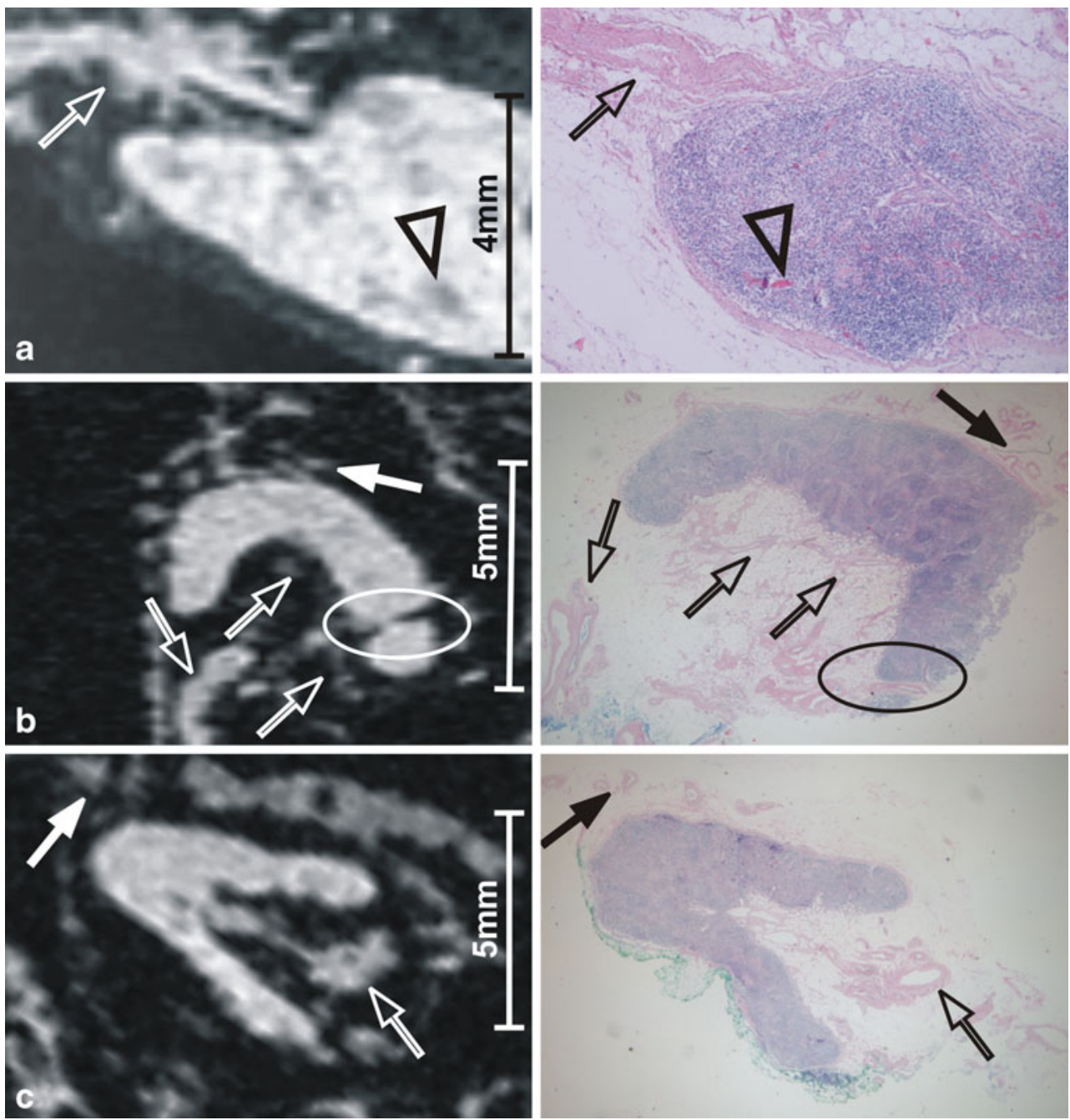
their $\mathrm{T} 2(33 \pm 2 \mathrm{~ms})$ and $\mathrm{T} 2 *(17 \pm 2 \mathrm{~ms})$ characteristics closely matched those of histologically-confirmed lymph nodes. Also anatomically, no other structures of corresponding size and shape are present in ALND specimens. For these particular nodes the mean T1 value could not be determined because they were not visualized on the (single slice) Look-Locker data set, and the ADC was of insufficient resolution for accurate ROI analysis. Three of the nine additional nodes however were pathologically confirmed lymph nodes. These nodes were only retrieved by the pathologist on the basis of the MRI, one after direct consultation of the MRI-based map for its precise location and two others after the specimen was more thoroughly defatted because the MRI-based map indicated more nodes should be present. The data therefore suggest that MRI has the potential to detect even more nodes than standard clinical pathological examination.

\section{Morphological characteristics of lymph nodes}

This study also indicates that not all benign nodes have a lipid rich medulla. Additionally, it is shown that size and shape of histologically-proven benign human lymph nodes can vary greatly. A fair correlation was found between size and the presence of a fatty centre. Larger lymph nodes were more likely to have a fatty centre than smaller lymph nodes. This is useful for their putative discrimination from malignant lymph nodes which are also, on average, larger, but often do not contain a fatty centre [17]. In the present study all nodes were histologically proven benign nodes, but this issue is addressed in an ongoing 7T study in lymph nodes derived from breast cancer patients. With respect to metastatic morphology; the roundness of nodes did not correlate with the presence of a fatty centre. In addition, while a diameter greater than 10 by $15 \mathrm{~mm}$ on transverse images is often used as a cutoff for the detection of metastatic nodes [8, 18-20], the diameter of the healthy nodes in this study -also measured in the transversal imaging plane- varied from 0.6 to $17.0 \mathrm{~mm}$ by 1.3 to $27.2 \mathrm{~mm}$.

Many efferent and afferent lymph- and blood vessels were visualized. Efferent blood vessels cannot always be discriminated from efferent lymph vessels on MRI. However, also pathologically, lymph vessels can only be differentiated from blood vessels with certainty if valves (lymph vessels) or erythrocytes (blood vessels) are present.

Several putative secondary B-cell follicles situated in the periphery of the nodes were observed. At pathology these nodes were normal reactive lymph nodes with multiple, activated B-cell follicles. The signal intensity of these follicles was not as hypo-intense as the hypointensity caused by the presence of erythrocytes (Fig. 6) which are found in blood vessels, nor was the shape of the structure similar to vessels. It is important to note that these areas of hypo-intensity in the nodal capsule on the (non-contrast-enhanced) $\mathrm{T} 1 \mathrm{~W}$ images are not metastatic involvement.

\section{Quantitative analysis}

Mean absolute T1, T2 and ADC values of ex vivo healthy human axillary nodes at $7 \mathrm{~T}$ were $944 \mathrm{~ms}, 32 \mathrm{~ms}$ and
Fig. 6 Two lymph nodes on 3D $\mathrm{T} 1 \mathrm{~W}$ images $(180 \mu \mathrm{m}$ isotropic resolution) and on matching H\&E stained histopathology images (1.5× magnification). Solid arrows: Afferent lymph vessels. Open arrows: Efferent vessels. Solid arrowhead: Loosely packed medullar structure. Thin black arrow: B-cell follicles. Open arrowhead: Intranodal dilated blood vessels. a A round shaped lymph node with a fatty centre. A vessel (encircled) traverses the capsule of the node. b A horseshoe shaped lymph node with a fatty centre
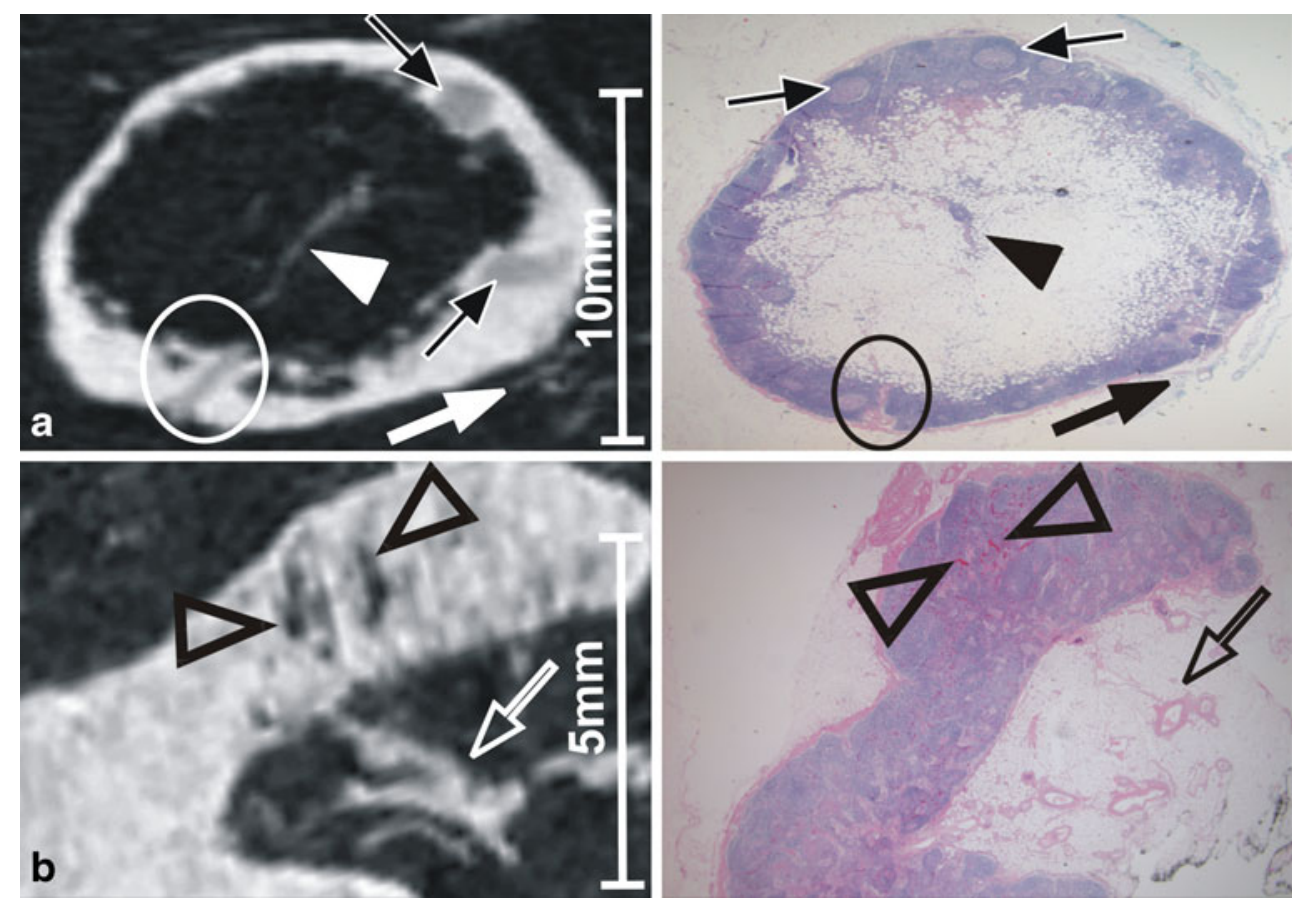
$0.39 \cdot 10^{-3} \mathrm{~mm}^{2} / \mathrm{s}$ respectively. Absolute $\mathrm{T} 2 *$ values were also determined. The high sensitivity of $\mathrm{T} 2 *$ to field heterogeneity often precludes the direct comparison of these values between different studies. But given the similar T2* values measured in this study, this effect seems to be reproducible in practice for the current set-up.

The quantitative measurements in the post-mortem specimens are influenced amongst others by fixation effects of formaldehyde and by temperature [15, 21-25]. Therefore these data can not be extrapolated directly to in vivo conditions. With respect to the temperature, the determined ex vivo values can be corrected as shown in the Materials and methods section; but correction for the effect of fixation on cellular metabolism is not possible. Especially for T1 values Tovi et al. showed a large dependency over time of T1 measurements for formaldehyde fixated tissue [25]. This effect was also visible in our study as shown in Fig. 2. Therefore, the reported absolute values cannot be directly compared to absolute values determined in vivo. For ex vivo studies, these measurements could nonetheless serve as a reference value for the future comparison of ex vivo healthy lymph nodes to metastatic nodes.

\section{Conclusion}

7T MRI of ex vivo human lymph nodes correlated well with pathology findings. MRI detected at least as many nodes in the ALND specimens as detected by standard clinical pathological examination and it visualized great structural detail such as efferent- and afferent vessels, intranodal capillary structures and several putative B-cell follicles. The detailed anatomical imaging, in addition to the quantitative analyses, of healthy nodes may support a better assessment of metastatic nodes in the future.

Open Access This article is distributed under the terms of the Creative Commons Attribution Noncommercial License which permits any noncommercial use, distribution, and reproduction in any medium, provided the original author(s) and source are credited.

\section{References}

1. Parkin DM, Bray F, Ferlay J et al (2005) Global cancer statistics, 2002. CA Cancer J Clin 55:74-108

2. Moore MP, Kinne DW (1997) Axillary lymphadenectomy: a diagnostic and therapeutic procedure. J Surg Oncol 66:2-6

3. Silverstein MJ, Skinner KA, Lomis TJ (2001) Predicting axillary nodal positivity in 2282 patients with breast carcinoma. World J Surg 25:767-772

4. Morrow M (1996) Role of axillary dissection in breast cancer management. Ann Surg Oncol 3:233-234

5. Shaw JH, Rumball EM (1990) Complications and local recurrence following lymphadenectomy. Br J Surg 77:760-764
6. Gartner R, Jensen MB, Nielsen J et al (2009) Prevalence of and factors associated with persistent pain following breast cancer surgery. JAMA 302:1985-1992

7. Kwee TC, Takahara T, Luijten PR et al (2009) ADC measurements of lymph nodes: inter- and intra-observer reproducibility study and an overview of the literature. Eur J Radiol. doi:10.1016/ j.ejrad.2009.03.026

8. de Bondt RB, Nelemans PJ, Bakers F et al (2009) Morphological MRI criteria improve the detection of lymph node metastases in head and neck squamous cell carcinoma: multivariate logistic regression analysis of MRI features of cervical lymph nodes. Eur Radiol 19:626-633

9. Harada T, Tanigawa N, Matsuki M et al (2007) Evaluation of lymph node metastases of breast cancer using ultrasmall superparamagnetic iron oxide-enhanced magnetic resonance imaging. Eur J Radiol 63:401-407

10. Luciani A, Pigneur F, Ghozali F et al (2009) Ex vivo MRI of axillary lymph nodes in breast cancer. Eur J Radiol 69:59-66

11. Dooms GC, Hricak H, Crooks LE et al (1984) Magnetic resonance imaging of the lymph nodes: comparison with CT. Radiology 153:719-728

12. Memarsadeghi M, Riedl CC, Kaneider A et al (2006) Axillary lymph node metastases in patients with breast carcinomas: assessment with nonenhanced versus uspio-enhanced MR imaging. Radiology 241:367-377

13. Benveniste H, Blackband S (2002) MR microscopy and high resolution small animal MRI: applications in neuroscience research. Prog Neurobiol 67:393-420

14. Hsu JJ, Lowe IJ (2004) Spin-lattice relaxation and a fast T1-map acquisition method in MRI with transient-state magnetization. J Magn Reson 169:270-278

15. Rieke V, Pauly KB (2008) MR thermometry. J Magn Reson Imaging 27:376-390

16. Földi M, Földi E (2006) Földi's textbook of lymphology for physicians and lymphedema therapists. In: Strössenreuther RHK, Kubik S (eds) 2nd edn. Elsevier GmbH, Munich pp 1-25

17. Abe H, Schmidt RA, Kulkarni K et al (2009) Axillary lymph nodes suspicious for breast cancer metastasis: sampling with USguided 14-gauge core-needle biopsy-clinical experience in 100 patients. Radiology 250:41-49

18. Holzapfel K, Duetsch S, Fauser C et al (2008) Value of diffusionweighted MR imaging in the differentiation between benign and malignant cervical lymph nodes. Eur J Radiol 72(3):381-387

19. Castelijns JA, van den Brekel MW (2002) Imaging of lymphadenopathy in the neck. Eur Radiol 12:727-738

20. Koc O, Paksoy Y, Erayman I et al (2007) Role of diffusion weighted MR in the discrimination diagnosis of the cystic and/or necrotic head and neck lesions. Eur J Radiol 62:205-213

21. Delannoy J, Chen CN, Turner R et al (1991) Noninvasive temperature imaging using diffusion MRI. Magn Reson Med 19:333-339

22. Shepherd TM, Flint JJ, Thelwall PE et al (2009) Postmortem interval alters the water relaxation and diffusion properties of rat nervous tissue-implications for MRI studies of human autopsy samples. Neuroimage 44:820-826

23. Shepherd TM, Thelwall PE, Stanisz GJ et al (2009) Aldehyde fixative solutions alter the water relaxation and diffusion properties of nervous tissue. Magn Reson Med 62:26-34

24. Thelwall PE, Shepherd TM, Stanisz GJ et al (2006) Effects of temperature and aldehyde fixation on tissue water diffusion properties, studied in an erythrocyte ghost tissue model. Magn Reson Med 56:282-289

25. Tovi M, Ericsson A (1992) Measurements of T1 and T2 over time in formalin-fixed human whole-brain specimens. Acta Radiol 33:400-404 